\title{
EMPIRICAL THERMOCHEMICAL VALUES FOR HIGHER ORDER OXIDES - AN UNCRITICAL CONSIDERATION
}

\author{
Mariolacos K. ${ }^{1}$ \\ Beethovenstr.38,D-37085 Göttingen, Germany.E-mail: kmariol@web.de
}

\begin{abstract}
This paper presents empirical thermochemical data for a number of higher order oxides and introduces the method employed for their calculations. ThIs method is based on experimentally measured data.

Key words: Thermochemistry, empirical values, oxides.
\end{abstract}

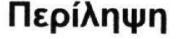

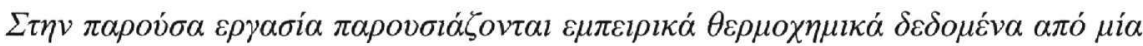

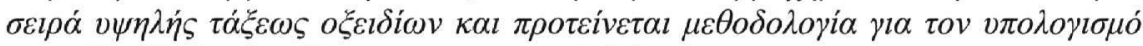

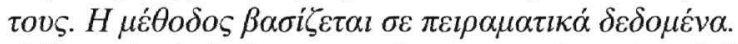

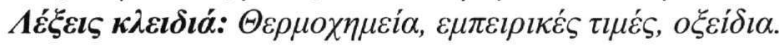

\section{Method}

The basis for the empirical calculation in the current study are the „uncritical" values given in Table 1, which lists the average thermochemical data for the binary oxides from Woods and Garrels (1987). The values for $\Delta \mathrm{G}^{\circ}{ }_{\text {cal }}$ and $\Delta \mathrm{H}^{\mathrm{o}}$ cal $[\mathrm{kJ} / \mathrm{mol}]$ respectively presented in Table 2, are calculated according the following principle: The sum of the values for the binary oxides are multiplied by the ratio of the sum corresponding to the atoms of the higher-order oxide over the sum corresponding to the atoms of the individual binary oxides. This yields the coefficients $\mathrm{C}_{\mathrm{G}}=$ $\Delta \mathrm{G}_{\text {exp }}^{\circ} / \Delta \mathrm{G}_{\text {cal }}^{\mathrm{C}}$ and $\mathrm{C}_{\mathrm{H}}=\Delta \mathrm{H}_{\text {exp }}^{\mathrm{o}} / \Delta \mathrm{H}_{\text {exp }}^{\mathrm{e}}$ respectively for both the ternary and quaternary oxides in the same table. For most of these calculations, the coefficients are approximately 1.10, with minimum and maximum values of 0.85 and 1.36 . The average for all coefficients is $\mathbf{C}_{\mathbf{G}, \mathbf{H}}=\mathbf{1 . 0 6 5 8}$.

In order to generate the empirical values for the oxides listed in Tables 3, 4 and 5, the quantities $\Delta \mathrm{G}_{\text {cal }}^{0}$ and $\Delta \mathrm{H}^{0}$ cal are multiplied by these coefficients.

For the current investigation only stoichiometric oxide compounds in a crystalline state were considered, i.e. carbonates, phosphates, nitrates etc. were not included. Additionally, neither compounds of other elements, e.g. chlorides, sulfides or fluorides, nor hydrates or hydroxides were considered. In accordance with the work of Woods and Garrels (1987), compounds of certain elements such as $\mathrm{Ga}, \mathrm{Gd}, \mathrm{In}, \mathrm{Nd}$ and $\mathrm{Tb}$ are also not included in the current study. All values given here are based on a temperature of $298.15 \mathrm{~K}$.

To clarify the proposed method, the following numerical examples are provided:

1. $\mathrm{Ba}_{2} \mathrm{Fe}_{2} \mathrm{O}_{5} \rightleftharpoons \mathrm{BaO}+\mathrm{Fe}_{2} \mathrm{O}_{3}$ 
$\Delta \mathrm{G}_{\text {cal }}^{\mathrm{o}}=[-521-743] \times[9 / 7]=-1625[\mathrm{~kJ} / \mathrm{mol}] ; \Delta \mathrm{G}_{\text {emp }}^{\mathrm{o}}=(-1625) \times 1.066=-1732[\mathrm{~kJ} / \mathrm{mol}]$

2. $\mathrm{Na}_{2} \mathrm{Mg}_{2} \mathrm{Si}_{2} \mathrm{O}_{7} \Rightarrow \mathrm{Na}_{2} \mathrm{O}+\mathrm{MgO}+\mathrm{SiO}_{2}$;

$\Delta \mathrm{G}_{\mathrm{cal}}^{\mathrm{o}}=[-377-569-853] \times[13 / 8]=-2923[\mathrm{~kJ} / \mathrm{mol}] ; \Delta \mathrm{G}_{\text {emp }}^{\mathrm{o}}=(-2923) \times 1.066=-3115[\mathrm{~kJ} / \mathrm{mol}]$

3. $\mathrm{PbCa}_{3} \mathrm{Zn}_{4} \mathrm{Si}_{4} \mathrm{O}_{16} \Rightarrow \mathrm{PbO}+\mathrm{CaO}+\mathrm{ZnO}+\mathrm{SiO}_{2}$;

$\Delta \mathrm{G}_{\text {cal }}^{\mathrm{o}}=[-189-604-321-853] \times[28 / 9]=-6119[\mathrm{~kJ} / \mathrm{mol}] ; \quad \Delta \mathrm{G}_{\mathrm{emp}}^{\mathrm{o}}=(-6119) \times 1.066=-6522$ $[\mathrm{kJ} / \mathrm{mol}]$

4. $\mathrm{CaYFeBe}_{2} \mathrm{Si}_{2} \mathrm{O}_{10} \Rightarrow \mathrm{CaO}+\mathrm{Y}_{2} \mathrm{O}_{3}+\mathrm{Fe}_{2} \mathrm{O}_{3}+\mathrm{BeO}+\mathrm{SiO}_{2}$;

$\Delta \mathrm{G}_{\text {cal }}^{\mathrm{o}}=[-604-1817-743-577-853] \times[17 / 17]=-4594[\mathrm{~kJ} / \mathrm{mol}] ; \Delta \mathrm{G}_{\mathrm{emp}}^{\mathrm{o}}=-4897[\mathrm{~kJ} / \mathrm{mol}]$

The values of $\Delta \mathrm{H}^{\circ}{ }_{\text {emp }}$ were calculated in a similar fashion.

Table 1 - Experimental thermochemical (average) values of binary oxides [1]

\begin{tabular}{|c|c|c|c|c|c|c|c|c|}
\hline \multicolumn{3}{|c|}{$\begin{array}{c}\text { Compound } \Delta \mathrm{G}_{\mathrm{f}}^{0} \Delta \mathrm{H}_{\mathrm{f}}^{0} \\
{[\mathrm{~kJ} / \mathrm{mol}]}\end{array}$} & \multicolumn{3}{|c|}{$\begin{array}{c}\text { Compound } \Delta \mathrm{G}_{\mathrm{f}}^{0} \quad \Delta \mathrm{H}_{\mathrm{f}}^{0} \\
{[\mathrm{~kJ} / \mathrm{mol}]}\end{array}$} & \multicolumn{3}{|c|}{$\begin{array}{c}\text { Compound } \Delta \mathrm{G}_{\mathrm{f}}^{0} \quad \Delta \mathrm{H}_{\mathrm{f}}^{0} \\
{[\mathrm{~kJ} / \mathrm{mol}]}\end{array}$} \\
\hline $\mathrm{AgO}$ & +11 & -25 & $\mathrm{Fe}_{2} \mathrm{O}_{3}$ & -743 & -824 & $\mathrm{SeO}_{2}$ & -173 & -227 \\
\hline $\mathrm{Ag}_{2} \mathrm{O}$ & -11 & -31 & $\mathrm{GeO}_{2}$ & -498 & -559 & $\mathrm{SiO}_{2}$ & -853 & -909 \\
\hline $\mathrm{Al}_{2} \mathrm{O}_{3}$ & -1578 & -1671 & $\mathrm{HgO}$ & -58 & -90 & $\mathrm{SnO}$ & -257 & -286 \\
\hline $\mathrm{As}_{2} \mathrm{O}_{3}$ & -580 & -659 & $\mathrm{MnO}$ & -363 & -385 & $\mathrm{SnO}_{2}$ & -519 & -581 \\
\hline $\mathrm{As}_{2} \mathrm{O}_{5}$ & -779 & -921 & $\mathrm{~K}_{2} \mathrm{O}$ & -320 & -362 & $\mathrm{SrO}$ & -564 & -594 \\
\hline $\mathrm{B}_{2} \mathrm{O}_{3}$ & -1191 & -1270 & $\mathrm{La}_{2} \mathrm{O}_{3}$ & -1726 & -1824 & $\mathrm{SrO}_{2}$ & -582 & -638 \\
\hline $\mathrm{BaO}$ & -521 & -549 & $\mathrm{Li}_{2} \mathrm{O}$ & -561 & -598 & $\mathrm{Ta}_{2} \mathrm{O}_{5}$ & -1926 & -2058 \\
\hline $\mathrm{BeO}$ & -577 & -606 & $\mathrm{MgO}$ & -569 & -601 & $\mathrm{TeO}_{2}$ & -270 & -322 \\
\hline $\mathrm{Bi}_{2} \mathrm{O}_{3}$ & -494 & -575 & $\mathrm{MnO}_{2}$ & -463 & -520 & $\mathrm{ThO}_{2}$ & -1168 & -1226 \\
\hline $\mathrm{CaO}$ & -604 & -635 & $\mathrm{Mn}_{2} \mathrm{O}_{3}$ & -884 & -961 & $\mathrm{TiO}_{2}$ & -887 & -940 \\
\hline $\mathrm{CdO}$ & -228 & -257 & $\mathrm{MoO}_{3}$ & -670 & -747 & $\mathrm{Ti}_{2} \mathrm{O}_{3}$ & -1433 & -1521 \\
\hline $\mathrm{CeO}_{2}$ & -1025 & -1089 & $\mathrm{Na}_{2} \mathrm{O}$ & -377 & -416 & $\mathrm{UO}_{2}$ & -1032 & -1085 \\
\hline $\mathrm{Ce}_{2} \mathrm{O}_{3}$ & -1707 & -1796 & $\mathrm{Nb}_{2} \mathrm{O}_{5}$ & -1765 & -1898 & $\mathrm{UO}_{3}$ & -1146 & -1223 \\
\hline $\mathrm{CoO}$ & -213 & -236 & $\mathrm{NiO}$ & -212 & -240 & $\mathrm{~V}_{2} \mathrm{O}_{3}$ & -1138 & -1227 \\
\hline $\mathrm{CrO}_{3}$ & & -589 & $\mathrm{P}_{2} \mathrm{O}_{5}$ & -1361 & -1506 & $\mathrm{~V}_{2} \mathrm{O}_{5}$ & -1419 & -1551 \\
\hline $\mathrm{Cr}_{2} \mathrm{O}_{3}$ & -1054 & -1136 & $\mathrm{PbO}$ & -189 & -218 & $\mathrm{WO}_{3}$ & -764 & -842 \\
\hline $\mathrm{Cs}_{2} \mathrm{O}$ & -297 & -332 & $\mathrm{PbO}_{2}$ & -217 & -276 & $\mathrm{~W}_{2} \mathrm{O}_{5}$ & -1284 & -1413 \\
\hline $\mathrm{Cs}_{2} \mathrm{O}_{3}$ & -360 & -465 & $\mathrm{Rb}_{2} \mathrm{O}$ & -291 & -333 & $\mathrm{Y}_{2} \mathrm{O}_{3}$ & -1817 & -1905 \\
\hline $\mathrm{CuO}$ & -127 & -156 & $\mathrm{Rb}_{2} \mathrm{O}_{3}$ & -387 & -488 & $\mathrm{Yb}_{2} \mathrm{O}_{3}$ & -1727 & -1815 \\
\hline $\mathrm{Cu}_{2} \mathrm{O}$ & -147 & -169 & $\mathrm{Sb}_{2} \mathrm{O}_{5}$ & -844 & -987 & $\mathrm{ZnO}$ & -321 & -350 \\
\hline $\mathrm{FeO}$ & -250 & -271 & $\mathrm{Sc}_{2} \mathrm{O}_{3}$ & -1819 & -1909 & $\mathrm{ZrO}_{2}$ & -1040 & -1099 \\
\hline
\end{tabular}

Table 2 - Experimental and calculated data for $\Delta \mathbf{G}^{0}{ }_{\exp }, \Delta \mathrm{G}^{0}$ cal, $\Delta \mathbf{H}_{\text {exp }}^{0}, \Delta \mathbf{H}_{\text {cal }}^{0}$ with coefficients $C_{G}=\Delta G_{\text {exp }}^{0} / \Delta G_{\text {cal }}^{0}$ and $C_{H}=\Delta H_{\text {exp }}^{0} / \Delta H^{0}{ }_{\text {cal }}$ respectively

a. Ternary oxides

\begin{tabular}{|c|c|c|c|c|c|c|}
\hline Compound & \multicolumn{2}{|c|}{$[\mathrm{kJ} / \mathrm{mol}]$} & $\mathrm{C}_{\mathrm{G}}$ & $\Delta H^{0}{ }_{\text {exp }}$ & $\begin{array}{l}\Delta \mathrm{H}_{\mathrm{cal}}^{\mathrm{a}} \\
\mathrm{mol}]\end{array}$ & $\mathrm{C}_{\mathrm{H}}$ \\
\hline $\mathrm{AgMoO}_{4}$ & -822 & -659 & 1.25 & & & \\
\hline $\mathrm{Ag}_{2} \mathrm{CrO}_{4}$ & & & & -731 & -620 & 1.18 \\
\hline $\mathrm{Ag}_{2} \mathrm{MoO}_{4}$ & -749 & -681 & 1.10 & -839 & -778 & 1.08 \\
\hline $\mathrm{Ag}_{2} \mathrm{WO}_{4}$ & -852 & -775 & 1.10 & -925 & -873 & 1.06 \\
\hline $\mathrm{Al}_{2} \mathrm{SiO}_{5}$ & -2441 & -2431 & 1.00 & -2589 & -2580 & 1.00 \\
\hline $\mathrm{Al}_{2} \mathrm{Si}_{2} \mathrm{O}_{7}$ & -3143 & -3343 & 0.94 & -3346 & -3547 & 0.94 \\
\hline
\end{tabular}




\begin{tabular}{|c|c|c|c|c|c|c|}
\hline $\mathrm{Al}_{6} \mathrm{Si}_{2} \mathrm{O}_{13}$ & -6434 & -6381 & 1.01 & -6815 & -6772 & 1.01 \\
\hline $\mathrm{BaMoO}_{4}$ & -1442 & -1191 & 1.21 & -1548 & -1296 & 1.19 \\
\hline $\mathrm{BaWO}_{4}$ & -1546 & -1285 & 1.20 & -1681 & -1391 & 1.21 \\
\hline $\mathrm{BaSiO}_{3}$ & -1537 & -1374 & 1.12 & -1619 & -1458 & 1.11 \\
\hline $\mathrm{BaSi}_{2} \mathrm{O}_{5}$ & -2403 & -2198 & 1.09 & -2543 & -2333 & 1.09 \\
\hline $\mathrm{Ba}_{2} \mathrm{SiO}_{3}$ & -1969 & -1649 & 1.19 & -2079 & -1750 & 1.19 \\
\hline $\mathrm{Ba}_{2} \mathrm{SiO}_{4}$ & -2160 & -1924 & 1.12 & -2279 & -2041 & 1.12 \\
\hline $\mathrm{Ba}_{2} \mathrm{Si}_{3} \mathrm{O}_{8}$ & -3948 & -3572 & 1.10 & -4176 & -3791 & 1.10 \\
\hline $\mathrm{Ba}_{3} \mathrm{SiO}_{5}$ & -2858 & -2473 & 1.16 & -3002 & -2624 & 1.14 \\
\hline $\mathrm{BeAl}_{2} \mathrm{O}_{4}$ & -2178 & -2155 & 1.01 & -2300 & -2277 & 1.01 \\
\hline $\mathrm{BeSiO}_{3}$ & -1454 & -1430 & 1.02 & -1540 & -1515 & 1.02 \\
\hline $\mathrm{Be}_{2} \mathrm{SiO}_{4}$ & -2038 & -2002 & 1.02 & -2153 & -2121 & 1.01 \\
\hline $\mathrm{CaFe}_{2} \mathrm{O}_{4}$ & -1413 & -1347 & 1.05 & -1520 & -1459 & 1.04 \\
\hline $\mathrm{Ca}_{2} \mathrm{Fe}_{2} \mathrm{O}_{5}$ & -2000 & -1732 & 1.15 & -2134 & -1876 & 1.14 \\
\hline $\mathrm{CaMoO}_{4}$ & -1440 & -1274 & 1.13 & -1547 & -1382 & 1.12 \\
\hline $\mathrm{CaSiO}_{3}$ & -1547 & -1457 & 1.06 & -1632 & -1544 & 1.06 \\
\hline $\mathrm{Ca}_{2} \mathrm{SiO}_{4}$ & -2196 & -2040 & 1.08 & -2312 & -2162 & 1.07 \\
\hline $\mathrm{Ca}_{3} \mathrm{SiO}_{5}$ & -2785 & -2623 & 1.06 & -2931 & -2779 & 1.05 \\
\hline $\mathrm{Ca}_{3} \mathrm{Si}_{2} \mathrm{O}_{7}$ & -3759 & -3497 & 1.07 & -3960 & -3706 & 1.07 \\
\hline $\mathrm{CaWO}_{4}$ & -1538 & -1368 & 1.12 & -1643 & -1477 & 1.11 \\
\hline $\mathrm{CdSiO}_{3}$ & -1104 & -1081 & 1.02 & -1188 & -1166 & 1.02 \\
\hline $\mathrm{FeAl}_{2} \mathrm{O}_{4}$ & -1864 & -1828 & 1.02 & -1979 & -1942 & 1.02 \\
\hline $\mathrm{FeCr}_{2} \mathrm{O}_{4}$ & -1358 & -1304 & 1.04 & -1459 & -1407 & 1.04 \\
\hline $\mathrm{FeMoO}_{4}$ & -977 & -920 & 1.06 & -1076 & -1018 & 1.06 \\
\hline $\mathrm{FeSiO}_{3}$ & -1119 & -1103 & 1.01 & -1193 & -1180 & 1.01 \\
\hline $\mathrm{Fe}_{2} \mathrm{SiO}_{4}$ & -1375 & -1544 & 0.89 & -1475 & -1652 & 0.89 \\
\hline $\mathrm{FeTiO}_{3}$ & -1159 & -1137 & 1.02 & -1236 & -1211 & 1.02 \\
\hline $\mathrm{FeWO}_{4}$ & -1061 & -1014 & 1.05 & -1161 & -1113 & 1.04 \\
\hline $\mathrm{K}_{2} \mathrm{SiO}_{3}$ & -1465 & -1173 & 1.25 & -1553 & -1271 & 1.22 \\
\hline $\mathrm{K}_{2} \mathrm{Si}_{2} \mathrm{O}_{5}$ & -2335 & -1759 & 1.33 & -2483 & -1906 & 1.30 \\
\hline $\mathrm{K}_{2} \mathrm{Si}_{4} \mathrm{O}_{9}$ & -4085 & -2932 & 1.39 & -4330 & -3177 & 1.36 \\
\hline $\mathrm{LiAlO}_{2}$ & -1128 & -1069 & 1.05 & -1190 & -1134 & 1.05 \\
\hline $\mathrm{Li}_{2} \mathrm{SiO}_{3}$ & -1553 & -1414 & 1.10 & -1640 & -1507 & 1.09 \\
\hline $\mathrm{Li}_{2} \mathrm{Si}_{2} \mathrm{O}_{5}$ & -2396 & -2121 & 1.13 & -2540 & -2260 & 1.12 \\
\hline $\mathrm{MgAl}_{2} \mathrm{O}_{4}$ & -2179 & -2147 & 1.01 & -2304 & -2272 & 1.01 \\
\hline $\mathrm{MgCr}_{2} \mathrm{O}_{4}$ & -1669 & -1623 & 1.03 & -1773 & -1737 & 1.02 \\
\hline $\mathrm{MgFe}_{2} \mathrm{O}_{4}$ & -1326 & -1312 & 1.01 & -1437 & -1425 & 1.01 \\
\hline $\mathrm{MgSiO}_{3}$ & -1461 & -1422 & 1.03 & -1549 & -1510 & 1.02 \\
\hline $\mathrm{Mg}_{2} \mathrm{SiO}_{4}$ & -2055 & -1991 & 1.03 & -2175 & -2114 & 1.03 \\
\hline $\mathrm{MgTiO}_{3}$ & -1484 & -1456 & 1.02 & -1572 & -1541 & 1.02 \\
\hline $\mathrm{MnSiO}_{3}$ & -1241 & -1216 & 1.02 & -1320 & -1294 & 1.02 \\
\hline $\mathrm{Mn}_{2} \mathrm{SiO}_{4}$ & -1631 & -1702 & 0.96 & -1729 & -1812 & 0.95 \\
\hline $\mathrm{MnWO}_{4}$ & -1204 & -1127 & 1.07 & -1305 & -1227 & 1.06 \\
\hline $\mathrm{Na}_{2} \mathrm{SiO}_{3}$ & -1468 & -1230 & 1.19 & -1560 & -1325 & 1.18 \\
\hline $\mathrm{Na}_{2} \mathrm{UO}_{4}$ & -1773 & -1523 & 1.16 & -1959 & -1639 & 1.19 \\
\hline $\mathrm{NiAl}_{2} \mathrm{O}_{4}$ & -1819 & -1790 & 1.02 & -1928 & -1911 & 1.01 \\
\hline $\mathrm{NiFe}_{2} \mathrm{O}_{4}$ & -972 & -955 & 1.02 & -1080 & -1064 & 1.01 \\
\hline $\mathrm{NiSiO}_{3}$ & -1128 & -1065 & 1.06 & & & \\
\hline $\mathrm{Ni}_{2} \mathrm{SiO}_{4}$ & -1294 & -1491 & 0.87 & -1408 & -1609 & 0.87 \\
\hline $\mathrm{PbMoO}_{4}$ & -956 & -859 & 1.11 & -1071 & -965 & 1.11 \\
\hline $\mathrm{PbSiO}_{3}$ & -1061 & -1042 & 1.02 & -1134 & -1129 & 1.00 \\
\hline $\mathrm{Pb}_{2} \mathrm{SiO}_{4}$ & -1253 & -1459 & 0.86 & -1336 & -1578 & 0.85 \\
\hline $\mathrm{PbWO}_{4}$ & -1020 & -953 & 1.07 & -1122 & -1060 & 1.06 \\
\hline
\end{tabular}




\begin{tabular}{|lcccccc|}
$\mathrm{SrSiO}_{3}$ & -1560 & -1417 & 1.10 & -1634 & -1503 & 1.09 \\
$\mathrm{Sr}_{2} \mathrm{SiO}_{4}$ & -2212 & -1984 & 1.11 & -2313 & -2104 & 1.10 \\
$\mathrm{Sr}_{3} \mathrm{SiO}_{5}$ & -2887 & -2551 & 1.13 & -3001 & -2705 & 1.11 \\
$\mathrm{SrWO}_{4}$ & -1538 & -1328 & 1.16 & -1654 & -1436 & 1.15 \\
$\mathrm{USiO}_{4}$ & -1891 & -1885 & 1.00 & -2000 & -1994 & 1.00 \\
$\mathrm{ZnAl}_{2} \mathrm{O}_{4}$ & & & & -2065 & -2021 & 1.02 \\
$\mathrm{ZnSiO}_{3}$ & -1175 & -1174 & 1.00 & -1265 & -1259 & 1.00 \\
$\mathrm{Zn}_{2} \mathrm{SiO}_{4}$ & -1525 & -1644 & 0.93 & -1638 & -1763 & 0.93 \\
$\mathrm{Zn}_{2} \mathrm{TiO}_{4}$ & -1535 & -1691 & 0.91 & -1649 & -1806 & 0.91 \\
$\mathrm{ZnWO}_{4}$ & -1124 & -1085 & 1.04 & -1233 & -1192 & 1.03 \\
$\mathrm{ZrSiO}_{4}$ & -1915 & -1893 & 1.01 & -2028 & -2008 & 1.01 \\
\hline
\end{tabular}

\section{b. Quaternary oxides}

\begin{tabular}{|c|c|c|c|c|c|c|}
\hline Compound & \multicolumn{2}{|c|}{$[\mathrm{kJ} / \mathrm{mol}]$} & $\mathrm{C}_{\mathrm{G}}$ & $\begin{array}{r}\Delta \mathrm{H}^{\mathrm{o}} \\
\quad[\mathrm{k} \\
\end{array}$ & $\begin{array}{l}\Delta \mathrm{H}^{\circ} \mathrm{cal} \\
\mathrm{ol}]\end{array}$ & $\mathrm{C}_{\mathrm{H}}$ \\
\hline $\mathrm{CaAl}_{2} \mathrm{SiO}_{6}$ & -3122 & -3035 & 1.03 & -3293 & -3215 & 1.02 \\
\hline $\mathrm{CaAl}_{2} \mathrm{Si}_{2} \mathrm{O}_{8}$ & -3999 & -3945 & 1.01 & -4226 & -4179 & 1.01 \\
\hline $\mathrm{Ca}_{2} \mathrm{Al}_{2} \mathrm{SiO}_{7}$ & -3791 & -3642 & 1.04 & -3989 & -3858 & 1.03 \\
\hline $\mathrm{Ca}_{3} \mathrm{Al}_{2} \mathrm{Si}_{3} \mathrm{O}_{12}$ & -6277 & -6070 & 1.03 & -6639 & -6430 & 1.03 \\
\hline $\mathrm{CaFeSi}_{2} \mathrm{O}_{6}$ & -2676 & -2438 & 1.10 & -2840 & -2593 & 1.09 \\
\hline $\mathrm{Ca}_{3} \mathrm{Fe}_{2} \mathrm{Si}_{3} \mathrm{O}_{12}$ & -5416 & -4400 & 1.23 & -5760 & -4736 & 1.22 \\
\hline $\mathrm{CaMgSiO}_{4}$ & -2143 & -2026 & 1.06 & -2262 & -2145 & 1.05 \\
\hline $\mathrm{CaMgSi}_{2} \mathrm{O}_{6}$ & -3032 & -2894 & 1.05 & -3206 & -3064 & 1.05 \\
\hline $\mathrm{Ca}_{2} \mathrm{MgSi}_{2} \mathrm{O}_{7}$ & -3678 & -3473 & 1.06 & -3875 & -3677 & 1.05 \\
\hline $\mathrm{CaTiSiO}_{5}$ & -2455 & -2344 & 1.05 & -2590 & -2484 & 1.04 \\
\hline $\mathrm{Fe}_{2} \mathrm{Al}_{4} \mathrm{Si}_{5} \mathrm{O}_{18}$ & -7961 & -7775 & 1.02 & -8450 & -8268 & 1.02 \\
\hline $\mathrm{Fe}_{3} \mathrm{Al}_{2} \mathrm{Si}_{3} \mathrm{O}_{12}$ & -4970 & -5362 & 0.93 & -5302 & -5702 & 0.93 \\
\hline $\mathrm{FeMgSi}_{2} \mathrm{O}_{6}$ & -2593 & -2388 & 1.08 & -2756 & -2544 & 1.08 \\
\hline $\mathrm{KAlSiO}_{4}$ & -2000 & -1751 & 1.14 & -2115 & -1872 & 1.13 \\
\hline $\mathrm{KAlSi}_{2} \mathrm{O}_{6}$ & -2866 & -2501 & 1.14 & -3028 & -2674 & 1.13 \\
\hline $\mathrm{KAlSi}_{3} \mathrm{O}_{8}$ & -3729 & -3251 & 1.15 & -3960 & -3477 & 1.14 \\
\hline $\mathrm{LiAlSiO}_{4}$ & -2007 & -1904 & 1.05 & -2121 & -2022 & 1.05 \\
\hline $\mathrm{LiAlSi}_{2} \mathrm{O}_{6}$ & -2868 & -2720 & 1.05 & -3038 & -2889 & 1.05 \\
\hline $\mathrm{Mg}_{2} \mathrm{Al}_{4} \mathrm{SiO}_{10}$ & -4982 & -5100 & 0.98 & -5278 & -5408 & 0.97 \\
\hline $\mathrm{Mg}_{2} \mathrm{Al}_{4} \mathrm{Si}_{5} \mathrm{O}_{18}$ & -8666 & -8700 & 1.00 & -9161 & -9225 & 0.99 \\
\hline $\mathrm{Mg}_{3} \mathrm{Al}_{2} \mathrm{Si}_{3} \mathrm{O}_{12}$ & -5949 & -6000 & 0.99 & -6275 & -6362 & 0.99 \\
\hline $\mathrm{NaAlSiO}_{4}$ & -1970 & -1787 & 1.10 & -2085 & -1906 & 1.09 \\
\hline $\mathrm{NaAlSi}_{2} \mathrm{O}_{6}$ & -2833 & -2553 & 1.11 & -3023 & -2724 & 1.11 \\
\hline $\mathrm{NaAlSi}_{3} \mathrm{O}_{8}$ & -3704 & -3318 & 1.12 & -3924 & -3541 & 1.11 \\
\hline
\end{tabular}

\section{Comments}

The method outlined here provides an estimation and is intended only as a first approximation in those cases where, due to lack of thermochemical data, exact calculation of mineralogical processes is not possible. In these cases, an experimental determination of the unknown quantities should be undertaken. However, it should be not forgotten that the production of pure stoichiometric compounds and the experimental determination of there thermochemical data are not simple procedures.

Closer examination of Tables $\mathbf{2 a}$ and $\mathbf{2} \mathbf{b}$ reveals that the coefficients $\mathbf{C}_{\mathbf{G}}$ and $\mathbf{C}_{\mathbf{H}}$ for potassium compounds have a higher average value, while magnesium and zinc compounds exhibit an average 
value lower than 1.06 (the average for all compounds, as stated above). Thus, the empirical values for each group of elements can be determined individually.

The compounds in Tables 3, 4, 5 and $\mathbf{6}$ were taken either from the Mineralogical Tables compiled by Strunz (1977) or from the literature.

A similar study of the free-enthalpy changes for sulfides was published by Barton and Skinner (1967).

Table 3 - Empirical data of $\Delta G^{0}{ }_{\text {emp }}$ and $\Delta \mathbf{H}^{0}{ }_{\text {emp }}$ for ternary oxides

\begin{tabular}{|c|c|c|c|c|c|c|c|c|}
\hline \multirow{2}{*}{$\begin{array}{l}\text { Compound } \\
\mathrm{Al}_{2} \mathrm{ZnO}_{4}\end{array}$} & \multicolumn{2}{|c|}{$\begin{array}{c}\Delta \mathrm{G}_{\mathrm{emp}}^{\mathrm{o}} \Delta \mathrm{H}_{\mathrm{emp}}^{\mathrm{o}} \\
{[\mathrm{kJ} / \mathrm{mol}]}\end{array}$} & \multicolumn{3}{|c|}{$\begin{array}{cc}\text { Compound } & \Delta \mathrm{G}_{\text {emp }}^{\mathrm{o}} \Delta \mathrm{H}^{\mathrm{o}}{ }_{\mathrm{emp}} \\
{[\mathrm{kJ} / \mathrm{mol}]}\end{array}$} & \multirow{2}{*}{$\frac{\text { Compound }}{\mathrm{Pb}_{3} \mathrm{~V}_{2} \mathrm{O}_{8}}$} & \multicolumn{2}{|c|}{$\begin{array}{c}\Delta \mathrm{G}^{\mathrm{o}} \mathrm{emp} \Delta \mathrm{H}^{\mathrm{o}} \mathrm{emp} \\
{[\mathrm{kJ} / \mathrm{mol}]}\end{array}$} \\
\hline & -2024 & -2154 & $\mathrm{LaVO}_{4}$ & -1676 & -1798 & & -2476 & -2723 \\
\hline $\mathrm{Ba}_{2} \mathrm{Fe}_{2} \mathrm{O}_{5}$ & -1732 & -1881 & $\mathrm{Li}_{2} \mathrm{Ge}_{7} \mathrm{O}_{15}$ & -4515 & -4932 & $\mathrm{SbFeO}_{4}$ & -845 & -964 \\
\hline $\mathrm{BaTiO}_{3}$ & -1501 & -1587 & $\mathrm{Li}_{2} \mathrm{SiO}_{3}$ & -1507 & -1606 & $\mathrm{Sc}_{2} \mathrm{Si}_{2} \mathrm{O}_{7}$ & -3916 & -4130 \\
\hline $\mathrm{BaSi}_{2} \mathrm{O}_{5}$ & -2343 & -2486 & $\mathrm{Li}_{2} \mathrm{Si}_{2} \mathrm{O}_{5}$ & -2260 & -2409 & $\mathrm{ScTaO}_{4}$ & -1995 & -2113 \\
\hline $\mathrm{BaZrO}_{3}$ & -1664 & -1756 & $\mathrm{Li}_{4} \mathrm{SiO}_{4}$ & -2260 & -2409 & $\mathrm{SnPb}_{2} \mathrm{O}_{4}$ & -1056 & -1193 \\
\hline $\mathrm{Bi}_{4} \mathrm{Si}_{3} \mathrm{O}_{12}$ & -3409 & -3756 & $\mathrm{Li}_{2} \mathrm{WO}_{4}$ & -1412 & -1535 & $\mathrm{Sn}_{2} \mathrm{Ta}_{2} \mathrm{O}_{7}$ & -2843 & -3053 \\
\hline $\mathrm{Bi}_{2} \mathrm{Sn}_{2} \mathrm{O}_{7}$ & -1485 & -1693 & $\mathrm{Mg}_{2} \mathrm{~B}_{2} \mathrm{O}_{5}$ & -2412 & -2564 & $\mathrm{SrAl}_{12} \mathrm{O}_{19}$ & -10436 & -11035 \\
\hline $\mathrm{BiVO}_{4}$ & -1019 & -1133 & $\mathrm{Mg}_{3} \mathrm{~B}_{2} \mathrm{O}_{6}$ & -2948 & -3133 & $\mathrm{SrFe}_{12} \mathrm{O}_{19}$ & -6368 & -6908 \\
\hline $\mathrm{CaB}_{2} \mathrm{O}_{4}$ & -1913 & -2030 & $\mathrm{MgGeO}_{3}$ & -1137 & -1236 & $\mathrm{SrB}_{2} \mathrm{O}_{4}$ & -1870 & -1987 \\
\hline $\mathrm{CaGeO}_{3}$ & -1174 & -1273 & $\mathrm{Mg}_{2} \mathrm{GeO}_{4}$ & -1592 & -1731 & $\mathrm{SrTiO}_{3}$ & -1546 & -1635 \\
\hline $\mathrm{Ca}_{2} \mathrm{GeO}_{4}$ & -1644 & -1782 & $\mathrm{MgNb}_{2} \mathrm{O}_{6}$ & -2487 & -2663 & $\mathrm{SrZrO}_{3}$ & -1709 & -1804 \\
\hline $\mathrm{CaMn}_{2} \mathrm{O}_{4}$ & -1586 & -1701 & $\mathrm{MgSb}_{2} \mathrm{O}_{6}$ & -1506 & -1692 & $\mathrm{ThSiO}_{4}$ & -2154 & -2275 \\
\hline $\mathrm{Ca}_{2} \mathrm{Ta}_{2} \mathrm{O}_{7}$ & -3295 & -3507 & $\mathrm{MgTi}_{2} \mathrm{O}_{5}$ & -2483 & -2628 & $\mathrm{ThTi}_{2} \mathrm{O}_{6}$ & -3285 & -3463 \\
\hline $\mathrm{CeAsO}_{4}$ & -1325 & -1447 & $\mathrm{Mg}_{2} \mathrm{TiO}_{4}$ & -2172 & -2299 & $\mathrm{TiBO}_{3}$ & -1398 & -1487 \\
\hline $\mathrm{CeVO}_{4}$ & -1666 & -1783 & $\mathrm{MgWO}_{4}$ & -1421 & -1538 & $\mathrm{Ti}_{2} \mathrm{MgO}_{4}$ & -2134 & -2262 \\
\hline $\mathrm{Co}_{2} \mathrm{~B}_{2} \mathrm{O}_{5}$ & -1924 & -2063 & $\mathrm{MnGeO}_{3}$ & -918 & -1006 & $\mathrm{UMo}_{2} \mathrm{O}_{8}$ & -2851 & -3068 \\
\hline $\mathrm{Co}_{3} \mathrm{~B}_{2} \mathrm{O}_{6}$ & -2351 & -2523 & $\mathrm{Mn}_{2} \mathrm{GeO}_{4}$ & -1284 & -1409 & $\mathrm{UTe}_{3} \mathrm{O}_{8}$ & -2775 & -2999 \\
\hline $\mathrm{CoGeO}_{3}$ & -758 & -847 & $\mathrm{MnNb}_{2} \mathrm{O}_{6}$ & -2268 & -2433 & $\mathrm{UTi}_{2} \mathbf{O}_{6}$ & -3067 & -3237 \\
\hline $\mathrm{Co}_{2} \mathrm{SiO}_{4}$ & -1590 & -1708 & $\mathrm{MnSb}_{2} \mathrm{O}_{6}$ & -1286 & -1462 & $\mathrm{VBO}_{3}$ & -1241 & -1330 \\
\hline $\mathrm{CoWO}_{4}$ & -1041 & -1149 & $\mathrm{Mn}_{6} \mathrm{Sb}_{2} \mathrm{O}_{15}{ }^{5}$ & -3412 & -3737 & $\mathrm{~V}_{2} \mathrm{FeO}_{4}$ & -1479 & -1596 \\
\hline $\mathrm{Cr}_{2} \mathrm{CuO}_{4}$ & -1259 & -1377 & $\mathrm{Mn}_{7} \mathrm{SiO}_{12}{ }^{6}$ & -4476 & -4807 & $\mathrm{Y}_{3} \mathrm{Al}_{5} \mathrm{O}_{12}$ & -7237 & -7623 \\
\hline $\mathrm{Cr}_{2} \mathrm{MnO}_{4}$ & -1510 & -1621 & $\mathrm{MnTa}_{2} \mathrm{O}_{6}$ & -2440 & -2604 & $\mathrm{YAsO}_{4}$ & -1383 & -1506 \\
\hline $\mathrm{Cr}_{2} \mathrm{NiO}_{4}$ & -1349 & -1466 & $\mathrm{MnTiO}_{3}$ & -1332 & -1412 & $\mathrm{YBO}_{3}$ & -1603 & -1691 \\
\hline $\mathrm{CuAs}_{2} \mathrm{O}_{4}$ & -753 & -869 & $\mathrm{Mn}_{2} \mathrm{ZnO}_{4}$ & -1284 & -1397 & $\mathrm{YNbO}_{4}$ & -1909 & -2026 \\
\hline $\mathrm{CuFeO}_{2}{ }^{1}$ & -402 & -455 & $\mathrm{Na}_{2} \mathrm{GeO}_{3}$ & -932 & -1039 & $\mathrm{Y}_{2} \mathrm{Si}_{2} \mathrm{O}_{7}$ & -3912 & -4123 \\
\hline $\mathrm{CuFeO}_{2}{ }^{2}$ & -474 & -529 & $\mathrm{NaNbO}_{3}$ & -1141 & -1233 & $\mathrm{YTaO}_{4}$ & -1994 & -2111 \\
\hline $\mathrm{Fe}_{3} \mathrm{BO}_{5}{ }^{3}$ & -1746 & -1891 & $\mathrm{Na}_{2} \mathrm{Si}_{2} \mathrm{O}_{5}$ & -1966 & -2118 & $\mathrm{ZnCr}_{2} \mathrm{O}_{4}$ & -1465 & -1584 \\
\hline $\mathrm{FeGeO}_{3}$ & -797 & -885 & $\mathrm{NaTaO}_{3}$ & -1227 & -1318 & $\mathrm{ZnFe}_{2} \mathrm{O}_{4}$ & -1134 & -1251 \\
\hline $\mathrm{Fe}_{2} \mathrm{MnO}_{4}$ & -1179 & -1288 & $\mathrm{Na}_{2} \mathrm{U}_{2} \mathrm{O}_{7}$ & -2550 & -2745 & $\mathrm{Zn}_{2} \mathrm{GeO}_{4}$ & -1222 & -1357 \\
\hline $\mathrm{FeNbO}_{4}$ & -1336 & -1450 & $\mathrm{NiAs}_{2} \mathrm{O}_{4}$ & -844 & -958 & $\mathrm{ZnNb}_{2} \mathrm{O}_{6}$ & -2223 & -2396 \\
\hline $\mathrm{FeNb}_{2} \mathrm{O}_{6}$ & -2147 & -2312 & $\mathrm{NiWO}_{4}$ & -1040 & -1153 & $\mathrm{ZnSb}_{2} \mathrm{O}_{6}$ & -1242 & -1425 \\
\hline $\mathrm{FeSb}_{2} \mathrm{O}_{6}$ & -1166 & -1341 & $\mathrm{PbCu}_{6} \mathrm{O}_{8}$ & -1100 & -1381 & $\mathrm{ZnTa}_{2} \mathrm{O}_{6}$ & -2395 & -2566 \\
\hline $\mathrm{Fe}_{5} \mathrm{Si}_{3} \mathrm{O}_{12}{ }^{4}$ & -3935 & -4272 & $\mathrm{PbSeO}_{3}$ & -386 & -474 & $\mathrm{ZrTiO}_{4}$ & -2054 & -2173 \\
\hline $\mathrm{Fe}_{2} \mathrm{ZnO}_{4}$ & -1134 & -1251 & $\mathrm{PbSeO}_{4}$ & -416 & -536 & $\mathrm{Zr}_{5} \mathrm{Ti}_{7} \mathrm{O}_{24}$ & -12323 & -13039 \\
\hline $\mathrm{Hg}_{2} \mathrm{TeO}_{4}$ & -489 & -615 & $\mathrm{PbTiO}_{3}$ & -1147 & -1234 & $\mathrm{ZrV}_{2} \mathrm{O}_{7}$ & -2621 & -2824 \\
\hline $\mathrm{K}_{2} \mathrm{CrO}_{4}$ & & -1014 & $\mathrm{~Pb}_{2} \mathrm{~V}_{2} \mathrm{O}_{7}^{7}$ & -2094 & -2304 & $\mathrm{ZnWO}_{4}$ & -1156 & -1270 \\
\hline & & & $\mathrm{Pb}_{2} \mathrm{~V}_{2} \mathrm{O}_{7}^{8}$ & -1985 & -2203 & $\mathrm{ZrW}_{2} \mathrm{O}_{8}$ & -3021 & -3251 \\
\hline
\end{tabular}


Table 4 - Empirical data of $\Delta \mathbf{G}^{0}$ emp and $\Delta \mathbf{H}^{0}{ }_{\text {emp }}$ for quaternary oxides

\begin{tabular}{|c|c|c|c|c|c|c|c|c|}
\hline Compound & $\begin{array}{c}\Delta \mathrm{G}^{\mathrm{o}}{ }_{\mathrm{emp}} \\
{[\mathrm{kJ} / 1}\end{array}$ & $\begin{array}{l}\Delta \mathrm{H}_{\mathrm{emp}}^{\mathrm{o}} \\
\mathrm{nol}]\end{array}$ & Compound & $\begin{array}{r}\Delta \mathrm{G}_{\text {emp }}^{\mathrm{o}} \\
{[\mathrm{kJ} /}\end{array}$ & $\begin{array}{l}\Delta \mathrm{H}^{\mathrm{o}}{ }_{\mathrm{emp}} \\
\mathrm{mol}]\end{array}$ & Compound & $\begin{array}{r}\Delta \mathrm{G}^{\mathrm{o}}{ }_{\mathrm{emp}} \\
{[\mathrm{kJ} / \mathrm{T}}\end{array}$ & $\begin{array}{l}\Delta \mathrm{H}^{\circ}{ }_{\text {emp }} \\
\mathrm{nol}]\end{array}$ \\
\hline $\mathrm{Al}_{16} \mathrm{~B}_{6} \mathrm{Si}_{2} \mathrm{O}_{37}$ & -18113 & -19254 & $\mathrm{Ca}_{3} \mathrm{Mn}_{2} \mathrm{Si}_{4} \mathrm{O}_{14}$ & -5738 & -6140 & $\mathrm{LaBSiO}_{5}$ & -2473 & -2625 \\
\hline $\mathrm{Al}_{4} \mathrm{MgBeO}_{8}$ & -4516 & -4772 & $\mathrm{Ca}_{2} \mathrm{MnSi}_{2} \mathrm{O}_{8}$ & -3325 & -3575 & $\mathrm{LiAlSiO}_{4}$ & -2029 & -2155 \\
\hline $\mathrm{Al}_{2} \mathrm{Be}_{3} \mathrm{Si}_{6} \mathrm{O}_{18}$ & -9297 & -9847 & $\mathrm{CaNiSi}_{2} \mathrm{O}_{6}$ & -2541 & -2717 & $\mathrm{LiAlSi}_{2} \mathrm{O}_{6}$ & -2899 & -3079 \\
\hline $\mathrm{BaAl}_{2} \mathrm{Ge}_{2} \mathrm{O}_{8}$ & -3598 & -3851 & $\mathrm{CaSnB}_{2} \mathrm{O}_{6}$ & -2466 & -2650 & $\mathrm{LiAlSi}_{4} \mathrm{O}_{10}$ & -4638 & -4926 \\
\hline $\mathrm{BaAl}_{2} \mathrm{Si}_{2} \mathrm{O}_{8}$ & -4090 & -4336 & $\mathrm{CaSnSiO}_{5}$ & -2106 & -2265 & $\mathrm{Li}_{2} \mathrm{BeSiO}_{4}$ & -2122 & -2252 \\
\hline $\mathrm{BaBe}_{2} \mathrm{Si}_{2} \mathrm{O}_{7}$ & -3565 & -3771 & $\mathrm{Ca}_{3} \mathrm{Ti}_{2} \mathrm{Si}_{3} \mathrm{O}_{12}$ & -6160 & -6533 & $\mathrm{LiFeSi}_{2} \mathrm{O}_{6}$ & -2090 & -2258 \\
\hline $\mathrm{BaCa}_{2} \mathrm{Si}_{3} \mathrm{O}_{9}$ & -4518 & -4780 & $\mathrm{CaTiZr}_{3} \mathrm{O}_{9}$ & -4720 & -4987 & $\mathrm{LiSbWO}_{6}$ & -1486 & -1663 \\
\hline $\mathrm{BaCuSi}_{4} \mathrm{O}_{10}$ & -3657 & -3932 & $\mathrm{Ca}_{3} \mathrm{~V}_{2} \mathrm{Si}_{3} \mathrm{O}_{12}$ & -5531 & -5907 & $\mathrm{Li}_{3} \mathrm{ScMo}_{3} \mathrm{O}_{12}$ & -5147 & -5492 \\
\hline $\mathrm{BaFeSi}_{4} \mathrm{O}_{10}$ & -3956 & -4212 & $\mathrm{CaZn}_{2} \mathrm{Ti}_{8} \mathrm{O}_{19}$ & -8277 & -8793 & $\mathrm{LiScSiO}_{4}$ & -2192 & -2317 \\
\hline $\mathrm{BaSnSi}_{3} \mathrm{O}_{9}$ & -3531 & -3803 & $\mathrm{Ca}_{2} \mathrm{ZnSi}_{2} \mathrm{O}_{7}$ & -3248 & -3461 & $\mathrm{LiScSi}_{2} \mathrm{O}_{6}$ & -3132 & -3309 \\
\hline $\mathrm{BaTiGe}_{3} \mathrm{O}_{9}$ & -3554 & -3820 & $\mathrm{CaZrTi}_{2} \mathrm{O}_{7}$ & -3709 & -3919 & $\mathrm{Mg}_{2} \mathrm{AlBO}_{5}$ & -2668 & -2831 \\
\hline $\mathrm{BaTiSi}_{3} \mathrm{O}_{9}$ & -4217 & -4472 & $\mathrm{CaYAl}_{3} \mathrm{O}_{7}$ & -4262 & -4488 & $\mathrm{MgAl}_{2} \mathrm{SiO}_{6}$ & -3197 & -3390 \\
\hline $\mathrm{Ba}_{2} \mathrm{TiSi}_{2} \mathrm{O}_{8}$ & -3916 & -4153 & $\mathrm{Cd}_{3} \mathrm{Al}_{2} \mathrm{Si}_{3} \mathrm{O}_{12}$ & -5668 & -6047 & $\mathrm{Mg}_{2} \mathrm{Al}_{4} \mathrm{SiO}_{10}$ & -5436 & -5764 \\
\hline $\mathrm{eMg}_{2} \mathrm{Al}_{6} \mathrm{O}_{12}$ & -6774 & -7157 & $\mathrm{Cd}_{3} \mathrm{~V}_{2} \mathrm{Si}_{3} \mathrm{O}_{12}$ & -4730 & -5101 & $\mathrm{Mg}_{2} \mathrm{Al}_{4} \mathrm{Si}_{5} \mathrm{O}_{18}$ & -9272 & -9832 \\
\hline $\mathrm{eMg}_{3} \mathrm{Al}_{8} \mathrm{O}_{16}$ & -9033 & -9543 & $\mathrm{CeBSiO}_{5}$ & -2460 & -2607 & $\mathrm{Mg}_{2} \mathrm{Al}_{4} \mathrm{Si}_{11} \mathrm{O}_{30}$ & -15028 & -15935 \\
\hline $\mathrm{Ca}_{3} \mathrm{Al}_{2} \mathrm{Si}_{3} \mathrm{O}_{12}$ & -6469 & -6853 & $\mathrm{CeNbTiO}_{6}$ & -2787 & -2963 & $\mathrm{Mg}_{3} \mathrm{Al}_{2} \mathrm{Si}_{3} \mathrm{O}_{12}$ & -6395 & -6781 \\
\hline $\mathbf{a}_{3} \mathrm{Al}_{2} \mathrm{Si}_{15} \mathrm{O}_{36}$ & -18114 & -19189 & $\mathrm{Co}_{3} \mathrm{Al}_{2} \mathrm{Si}_{3} \mathrm{O}_{12}$ & -5636 & -6003 & $\mathrm{Mg}_{3} \mathrm{Cr}_{2} \mathrm{Si}_{3} \mathrm{O}_{12}$ & -5278 & -5640 \\
\hline $\mathrm{CaB}_{2} \mathrm{Si}_{2} \mathrm{O}_{8}$ & -3668 & -3899 & $\mathrm{CsFeSi}_{2} \mathrm{O}_{6}$ & -1834 & -2000 & $\mathrm{Mg}_{2} \mathrm{FeBO}_{5}$ & -2000 & -2254 \\
\hline $\mathrm{a}_{2} \mathrm{BeSi}_{2} \mathrm{O}_{7}$ & -3716 & -3928 & $\mathrm{Cs}_{3} \mathrm{Mn}_{3} \mathrm{~V}_{4} \mathrm{O}_{16}$ & -4803 & -5254 & $\mathrm{Mg}_{3} \mathrm{Fe}_{2} \mathrm{GeO}_{8}$ & -2701 & -2961 \\
\hline $\mathrm{aBi}_{2} \mathrm{Nb}_{2} \mathrm{O}_{9}$ & -3051 & -3312 & $\mathrm{Cs}_{3} \mathrm{ScSi}_{8} \mathrm{O}_{19}$ & -8917 & -9461 & $\mathrm{MgFeSiO}_{4}$ & -1782 & -1898 \\
\hline $\mathrm{a}_{3} \mathrm{Cr}_{2} \mathrm{Ge}_{3} \mathrm{O}_{12}$ & -4596 & -4967 & $\mathrm{CuZn}{ }_{2} \mathrm{As}_{2} \mathrm{O}_{8}$ & -1545 & -1794 & $\mathrm{Mg}_{3} \mathrm{Fe}_{2} \mathrm{Si}_{3} \mathrm{O}_{12}$ & -4615 & -4975 \\
\hline $\mathrm{Ca}_{3} \mathrm{Cr}_{2} \mathrm{Si}_{3} \mathrm{O}_{12}$ & -5352 & -5713 & $\mathrm{Fe}_{2} \mathrm{Al}_{4} \mathrm{Si}_{5} \mathrm{O}_{18}$ & -8286 & -8812 & $\mathrm{Mg}_{2} \mathrm{MnBO}_{5}$ & -2113 & -2264 \\
\hline $\mathrm{CaCuSi}_{4} \mathrm{O}_{10}$ & -3859 & -4142 & $\mathrm{Fe}_{3} \mathrm{Al}_{2} \mathrm{Si}_{3} \mathrm{O}_{12}$ & -5715 & -6077 & $\mathrm{MgScBO}_{4}$ & -2225 & -2350 \\
\hline $\mathrm{CaFeSiO}_{4}$ & -1819 & -1934 & $\mathrm{Fe}_{2} \mathrm{Ba}_{3} \mathrm{Ge}_{3} \mathrm{O}_{12}$ & -3756 & -4118 & $\mathrm{MnAl}_{2} \mathrm{Si}_{2} \mathrm{O}_{8}$ & -3871 & -4107 \\
\hline $\mathrm{CaFeSi}_{2} \mathrm{O}_{6}$ & -2599 & -2764 & $\mathrm{FeCoBO}_{4}$ & -1334 & -1448 & $\mathrm{Mn}_{2} \mathrm{Al}_{4} \mathrm{Si}_{5} \mathrm{O}_{18}$ & -8636 & -9164 \\
\hline $\mathrm{Ca}_{2} \mathrm{Fe}_{3} \mathrm{Si}_{3} \mathrm{O}_{12}{ }^{\mathrm{i}}$ & -4352 & -4684 & $\mathrm{Fe}_{3} \mathrm{Cr}_{2} \mathrm{Si}_{3} \mathrm{O}_{12}$ & -4598 & -4937 & $\mathrm{Mn}_{3} \mathrm{Al}_{2} \mathrm{Si}_{3} \mathrm{O}_{12}$ & -5956 & -6320 \\
\hline $\mathrm{CaK}_{2} \mathrm{As}_{2} \mathrm{O}_{7}$ & -1815 & -2044 & $\mathrm{Fe}_{2} \mathrm{MgTi}_{3} \mathrm{O}_{10}$ & -3749 & -4033 & $\mathrm{Mn}_{3} \mathrm{Cr}_{2} \mathrm{Si}_{3} \mathrm{O}_{12}$ & -4839 & -5180 \\
\hline $\mathrm{CaLa}_{4} \mathrm{Si}_{3} \mathrm{O}_{13}$ & -7124 & -7538 & $\mathrm{KAlGeO}_{4}$ & -1625 & -1757 & $\mathrm{Mn}_{3} \mathrm{Fe}_{2} \mathrm{Si}_{3} \mathrm{O}_{12}$ & -4176 & -4515 \\
\hline $\mathrm{CaMgAl}_{14} \mathrm{O}_{23}$ & -12705 & -13426 & $\mathrm{KAlTi}_{3} \mathrm{O}_{8}$ & -3507 & -3744 & $\mathrm{NaBSi}_{3} \mathrm{O}_{8}$ & -3049 & -3269 \\
\hline $\mathrm{CaMg}_{2} \mathrm{Al}_{16} \mathrm{O}_{27}$ & -14986 & -15836 & $\mathrm{KFeSiO}_{4}$ & -1299 & -1421 & $\mathrm{NaCrSi}_{2} \mathrm{O}_{6}$ & -2213 & -2384 \\
\hline $\mathrm{aMgGeO}_{4}$ & -1781 & -1913 & $\mathrm{KFeSi}_{2} \mathrm{O}_{6}$ & -1857 & -2029 & $\mathrm{NaFeGe}_{2} \mathrm{O}_{6}$ & -1568 & -1742 \\
\hline $\mathrm{a}_{3} \mathrm{MgSi}_{2} \mathrm{O}_{8}$ & -4319 & -4572 & $\mathrm{KFeSi}_{3} \mathrm{O}_{8}$ & -2413 & -2639 & $\mathrm{NaFeSi}_{2} \mathrm{O}_{6}$ & -1912 & -2082 \\
\hline $\mathrm{CaMnSiO}_{4}$ & -1940 & -2056 & $\mathrm{~K}_{2} \mathrm{TiSi}_{4} \mathrm{O}_{11}$ & -4391 & -4713 & $\mathrm{NaFe}_{6} \mathrm{Si}_{6} \mathrm{O}_{20}$ & -6014 & -6547 \\
\hline $\mathrm{CaMnSi}_{2} \mathrm{O}_{6}$ & -2771 & -2937 & $\mathrm{~K}_{2} \mathrm{ZrSi}_{3} \mathrm{O}_{9}$ & -3931 & -4210 & $\mathrm{Na}_{2} \mathrm{FeSi}_{2} \mathrm{O}_{6}$ & -2169 & -2338 \\
\hline $\mathrm{CaMn}_{4} \mathrm{Si}_{5} \mathrm{O}_{15}$ & -6928 & -7342 & $\mathrm{~K}_{2} \mathrm{ZrSi}_{6} \mathrm{O}_{15}$ & -6289 & -6736 & $\mathrm{NaLa}_{9} \mathrm{Ge}_{6} \mathrm{O}_{26}$ & -10584 & -11390 \\
\hline $\mathrm{Na}_{2} \mathrm{Mg}_{2} \mathrm{Si}_{2} \mathrm{O}_{7}$ & -3115 & -3336 & $\mathrm{Na}_{2} \mathrm{ZrSi}_{2} \mathrm{O}_{7}$ & -3226 & -3360 & $\mathrm{Rb}_{2} \mathrm{ZnSi}_{5} \mathrm{O}_{12}$ & -3903 & -4242 \\
\hline $\mathrm{NaMnSi}_{2} \mathrm{O}_{6}$ & -2048 & -2436 & $\mathrm{Ni}_{3} \mathrm{Al}_{2} \mathrm{SiO}_{8}$ & -3943 & -4208 & $\mathrm{Sc}_{2} \mathrm{Be}_{3} \mathrm{Si}_{6} \mathrm{O}_{18}$ & -10042 & -13229 \\
\hline $\mathrm{Na}_{3} \mathrm{Mn}_{5} \mathrm{Si}_{8} \mathrm{O}_{24}{ }^{\mathrm{iii}}$ & -8122 & -8759 & $\mathrm{PbAl}_{2} \mathrm{Si}_{2} \mathrm{O}_{8}$ & -3630 & -3876 & $\mathrm{SrAl}_{2} \mathrm{Si}_{2} \mathrm{O}_{8}$ & -4149 & -4397 \\
\hline $\mathrm{NaNbAl}_{2} \mathrm{O}_{6}$ & -2643 & -2832 & $\mathrm{~Pb}_{3} \mathrm{BiAs}_{3} \mathrm{O}_{12}$ & -2114 & -2479 & $\mathrm{SrCuSi}_{4} \mathrm{O}_{10}$ & -3761 & -4041 \\
\hline $\mathrm{NaSbBe}_{4} \mathrm{O}_{7}$ & -2076 & -2319 & $\mathrm{~Pb}_{3} \mathrm{BiV}_{3} \mathrm{O}_{12}$ & -3041 & -3390 & $\mathrm{Sr}_{2} \mathrm{~V}_{2} \mathrm{Si}_{4} \mathrm{O}_{14}^{\text {iv }}$ & -5658 & -5904 \\
\hline $\mathrm{NaScSi}_{2} \mathrm{O}_{6}$ & -2954 & -3133 & $\mathrm{PbCu}_{2} \mathrm{Se}_{3} \mathrm{O}_{9}$ & -1117 & -1373 & $\mathrm{YAl}_{3} \mathrm{~B}_{4} \mathrm{O}_{12}$ & -6517 & -6886 \\
\hline $\mathrm{Na}_{2} \mathrm{TiSi}_{2} \mathrm{O}_{7}$ & -3009 & -3219 & $\mathrm{~Pb}_{2} \mathrm{Fe}_{2} \mathrm{Si}_{2} \mathrm{O}_{9}$ & -2853 & -3118 & $\mathrm{YNbTiO}_{6}$ & -2857 & -3033 \\
\hline $\mathrm{Na}_{2} \mathrm{TiSi}_{4} \mathrm{O}_{11}$ & -4512 & -4828 & $\mathrm{~Pb}_{2} \mathrm{Mn}_{2} \mathrm{Si}_{2} \mathrm{O}_{9}$ & -3079 & -3338 & $\mathrm{Y}_{2} \mathrm{SiBe}_{2} \mathrm{O}_{7}$ & -4152 & -4374 \\
\hline $\mathrm{Na}_{2} \mathrm{Ti}_{2} \mathrm{Si}_{2} \mathrm{O}_{9}$ & -3760 & -4023 & $\mathrm{~Pb}_{8} \mathrm{MnSi}_{6} \mathrm{O}_{21}$ & -7701 & -8288 & YTiTaO $_{6}$ & -2961 & -3135 \\
\hline $\mathrm{Na}_{2} \mathrm{ZrSi}_{4} \mathrm{O}_{11}$ & -4839 & -5167 & $\mathrm{PbZnSiO}_{4}$ & -1453 & -1574 & $\mathrm{ZrK}_{2} \mathrm{Si}_{3} \mathrm{O}_{9}$ & -3931 & -4210 \\
\hline
\end{tabular}

i $\mathrm{Ca}_{2} \mathrm{Fe}^{2+} \mathrm{Fe}^{3+}{ }_{2} \mathrm{Si}_{3} \mathrm{O}_{12} ; \quad$ ii $\mathrm{NaFe}_{3}^{2+}{ }_{3} \mathrm{Fe}_{3}^{3+} \mathrm{Si}_{6} \mathrm{O}_{20} ; \quad$ iii $\mathrm{Na}_{3} \mathrm{Mn}_{3}^{3+} \mathrm{Mn}_{2}^{2+} \mathrm{Si}_{8} \mathrm{O}_{24} ; \quad$ iv $\mathrm{Sr}^{2+} \mathrm{Sr}^{4+} \mathrm{V}^{3+}{ }_{2} \mathrm{Si}_{4} \mathrm{O}_{14}$ 
Table 5 - Empirical data of $\Delta \mathbf{G}^{\mathbf{0}}{ }_{\mathrm{emp}}$ and $\Delta \mathbf{H}^{\mathbf{0}}{ }_{\mathrm{emp}}$ for quinary oxides

\begin{tabular}{|c|c|c|c|c|c|}
\hline \multirow{2}{*}{$\begin{array}{r}\text { Compound } \\
\mathbf{B a F e}_{\mathbf{2}} \mathbf{T i S i}_{\mathbf{2}} \mathbf{O}_{\mathbf{9}}\end{array}$} & \multicolumn{2}{|c|}{$\begin{array}{c}\Delta \mathrm{G}^{\mathrm{o}}{ }_{\mathrm{emp}} \Delta \mathrm{H}_{\mathrm{emp}}^{\mathrm{o}} \\
{[\mathrm{kJ} / \mathrm{mol}]}\end{array}$} & \multirow{2}{*}{$\begin{array}{c}\text { Compound } \\
\mathrm{Ca}_{3} \mathrm{Zr}_{2} \mathrm{Al}_{2} \mathrm{SiO}_{12}\end{array}$} & \multicolumn{2}{|c|}{$\begin{array}{c}\Delta \mathrm{G}^{\circ}{ }_{\mathrm{emp}} \Delta \mathrm{H}^{\circ}{ }_{\mathrm{emp}} \\
{[\mathrm{kJ} / \mathrm{mol}]}\end{array}$} \\
\hline & -4014 & -4266 & & -6681 & -7074 \\
\hline $\mathrm{BaMg}_{2} \mathrm{Al}_{6} \mathrm{Si}_{9} \mathrm{O}_{30}$ & -15011 & -15902 & $\mathrm{Ca}_{3} \mathrm{Zr}_{2} \mathrm{Fe}_{2} \mathrm{SiO}_{12}$ & -5313 & -5685 \\
\hline $\mathrm{BaNa}_{2} \mathrm{Al}_{4} \mathrm{Si}_{4} \mathrm{O}_{16}$ & -7369 & -7847 & $\mathrm{KNa}_{3} \mathrm{Al}_{4} \mathrm{Si}_{4} \mathrm{O}_{16}$ & -6668 & -7158 \\
\hline $\mathrm{BaSr}_{2} \mathrm{Mn}_{2} \mathrm{Si}_{4} \mathrm{O}_{14}$ & -5765 & -6155 & $\mathrm{KNa}_{3} \mathrm{Mg}_{4} \mathrm{Si}_{12} \mathrm{O}_{30}$ & -10266 & -11084 \\
\hline $\mathrm{CaAlFeSiO}_{6}$ & -2685 & -2870 & $\mathrm{Mg}_{2} \mathrm{Al}_{14} \mathrm{~B}_{4} \mathrm{Si}_{4} \mathrm{O}_{37}$ & -18164 & -19292 \\
\hline $\mathrm{CaCrAlSiO}_{6}$ & -2905 & -3092 & $\mathrm{Mg}_{3} \mathrm{Al}_{4} \mathrm{Si}_{3} \mathrm{BeO}_{16}$ & -8577 & -9082 \\
\hline $\mathrm{Ca}_{2} \mathrm{FeB}_{2} \mathrm{Si}_{2} \mathrm{O}_{10}$ & -4375 & -4657 & $\mathrm{MgCrAlSiO}_{6}$ & -2881 & -3067 \\
\hline $\mathrm{Ca}_{6} \mathrm{Mg}_{5} \mathrm{MnB}_{12} \mathrm{O}_{30}$ & -14268 & -15126 & $\mathrm{NaMg}_{2} \mathrm{CrSi}_{3} \mathrm{O}_{10}$ & -3976 & -4267 \\
\hline $\mathrm{CaMn}_{2} \mathrm{Be}_{3} \mathrm{Si}_{3} \mathrm{O}_{12}$ & -5961 & -6304 & $\mathrm{Na}_{2} \mathrm{BaTi}_{2} \mathrm{Si}_{4} \mathrm{O}_{14}$ & -5879 & -6271 \\
\hline $\mathrm{CaNa}_{2} \mathrm{Al}_{4} \mathrm{Si}_{4} \mathrm{O}_{16}$ & -7552 & -8037 & $\mathrm{Na}_{2} \mathrm{Fe}_{5} \mathrm{TiSi}_{6} \mathrm{O}_{20}$ & -7797 & -8354 \\
\hline $\mathrm{Ca}_{2} \mathrm{NaMg}_{2} \mathrm{As}_{3} \mathrm{O}_{12}$ & -3546 & -3618 & $\mathrm{PbCa}_{3} \mathrm{Zn}_{4} \mathrm{Si}_{4} \mathrm{O}_{16}$ & -6522 & -7003 \\
\hline $\mathrm{Ca}_{3} \mathrm{TiFeSi}_{3} \mathrm{O}_{12}$ & -5529 & -5872 & $\mathrm{SrMg}_{2} \mathrm{Al}_{6} \mathrm{Si}_{9} \mathrm{O}_{30}$ & -15194 & -16093 \\
\hline $\mathrm{Ca}_{3} \mathrm{Ti}_{2} \mathrm{Fe}_{2} \mathrm{SiO}_{12}$ & -5061 & -5424 & $\mathrm{Y}_{2} \mathrm{FeBe}_{2} \mathrm{Si}_{2} \mathrm{O}_{10}$ & -5280 & -5573 \\
\hline $\mathrm{CaZrBAl}_{9} \mathrm{O}_{18}$ & -9407 & -9965 & & & \\
\hline
\end{tabular}

Table 6 - Empirical data of $\Delta G^{0}$ emp and $\Delta H^{0}$ emp for senary oxides

\begin{tabular}{|c|c|c|}
\hline Compound & \multicolumn{2}{|c|}{$[\mathrm{kJ} / \mathrm{mol}]$} \\
\hline $\mathrm{CaMgFe}_{2} \mathrm{Al}_{2} \mathrm{SiO}_{10}$ & -4633 & -4945 \\
\hline $\mathrm{CaYFeBe}_{2} \mathrm{Si}_{2} \mathrm{O}_{10}$ & -4896 & -5200 \\
\hline $\mathrm{Na}_{4} \mathrm{BaTi}_{2} \mathrm{~B}_{2} \mathrm{Si}_{10} \mathrm{O}_{30}$ & -12497 & -13330 \\
\hline $\mathrm{Na}_{3} \mathrm{Mg}_{3} \mathrm{FeTiSi}{ }_{8} \mathrm{O}_{24}$ & -9136 & -9832 \\
\hline
\end{tabular}

\section{Acknowledgement}

The translation of the paper in English by Dr. John Balk, University of Kentucky, is gratefully acknowledged.

\section{References}

Barton, P.B., and Skinner, B.J., 1967. Sulfide mineral stabilities. In H.L. Barnes (ed.), Geochemistry of Hydrothermal Ore Deposits, 238-326pp. Holt, Rinehart \& Winston.

Strunz, H., 1977. Mineralogische Tabellen, Akademische Verlagsgesellschaft, Leipzig.

Woods, L.T., and Garrels, R., 1987. Thermodynamic Values at low Temperature for natural inorganic Materials: An uncritical Summary, Oxford University Press, New York, Oxford. 